Z Gerontol Geriat 2014 · 47:88-89

DOI 10.1007/s00391-014-0607-z

Online publiziert: 21. Februar 2014

(c) Springer-Verlag Berlin Heidelberg 2014
M. Gosch ${ }^{1}$ H.J. Heppner ${ }^{2,3}$

${ }^{1}$ Abteilung für Geriatrie und Innere Medizin, Hospital Hochzirl, Zirl

${ }^{2}$ Geriatrische Klinik, HELIOS Klinikum Schwelm

${ }^{3}$ Lehrstuhl Geriatrie, Universität Witten/Herdecke

\title{
Das perioperative Management der geriatrischen Patienten
}

\section{Eine Herausforderung, heute und in der Zukunft}

Vielen ist die Bedeutung des demographischen Wandels und dessen Auswirkungen auf das Gesundheitssystem bewusst. Mit unserem Themenschwerpunkt "perioperatives Management" möchten wir dennoch ein unseres Erachtens relativ neues Kapitel der geriatrischen Medizin beleuchten und auf dessen rasch zunehmende Bedeutung hinweisen. Im Rahmen der Entwicklung der Alterstraumatologie haben sich für die Geriatrie neue Herausforderungen in der täglichen Arbeit gezeigt. Das interdisziplinäre und interprofessionelle perioperative Management der geriatrischen Patienten wird zunehmend zu einem fixen Bestandteil der geriatrischen Medizin, wobei die operative Traumatologie per se nur einen Teil der operativen Eingriffe bei Hochbetagten abbildet. In den nächsten Jahren ist mit einer Verdoppelung älterer Notfallpatienten zu rechnen. Im Vergleich zur Gesamtbevölkerung nehmen Notfallpatienten über 70 Jahre etwa 4-mal so schnell zu [1]. Ähnlich verhält es sich mit der Entwicklung der Operationszahlen der letzten Jahre in Deutschland (• Abb. 1). Von 2007 bis 2012 stieg die Anzahl der Operationen in allen Altersgruppen um $18 \%$. In den Altersgruppen über 70 Jahre war der prozentuelle Zuwachs deutlich stärker ausgeprägt und erreicht ein Maximum in der Altersgruppe der 90-94-Jährigen mit einer Steigerung von $54 \%$ [2].
Der Durchschnitt liegt z. B. für das Einsetzen von Hüftprothesen in Deutschland mit 295 Eingriffen pro 100.000 Einwohner in der EU-Statistik weit vorn [3]. Leider findet sich im Bereich der perioperativen Betreuung der geriatrischen Patienten eine in der Geriatrie weitverbreitete Diskrepanz zwischen der hohen klinischen Relevanz und der geringen wissenschaftlichen Evidenz.

Für unseren Themenschwerpunkt haben wir uns auf unserer Meinung nach 6 wichtige Gebiete und Fragestellungen beschränkt. Gerade bei betagten Patienten kommt die Risiko-Nutzen-Abwägung und den sich daraus ergebenden Konsequenzen eine entscheidende Bedeutung zu. Der Beitrag von Beck et al. zur Risikostratifizierung geriatrischer Patienten bei nichtkardialen Eingriffen gibt einen Überblick über aktuelle Tools unter besonderer Berücksichtigung des geriatrischen Assessments und dessen praktische Umsetzung. In der Planung eines operativen Eingriffs kommt dem perioperativen Gerinnungsmanagement eine besondere Bedeutung zu. Aufgrund der häufigen Komorbiditäten ist im Gegensatz zu jüngeren Kollektiven der Anteil der Patienten mit einer vorstehenden gerinnungshemmenden Therapie groß, sei es die präventive Einnahme von Acetylsalicylsäure, eine orale Antikoagulation bei Vorhofflimmern oder nach einem thromboem- 


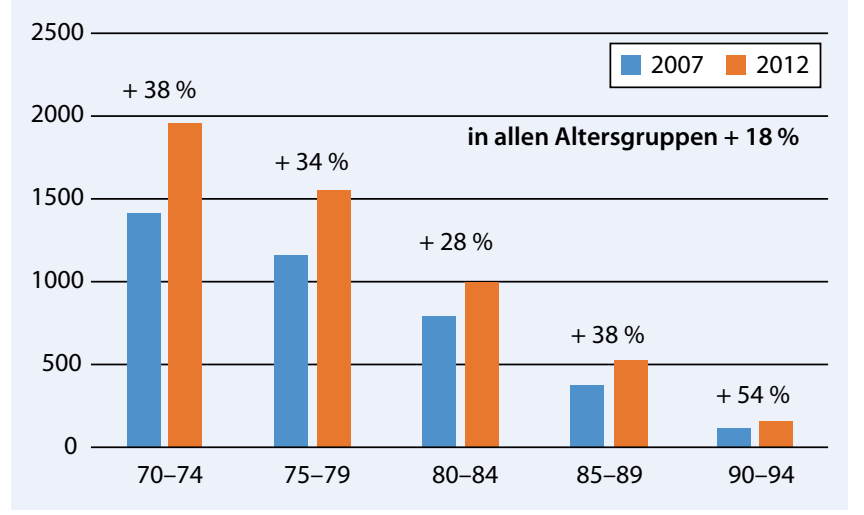

Abb. $1<$ Operationszahlen (in Tausenden) in Deutschland 2007 und 2012 nach Altersgruppen bolischen Ereignis. Die Evidenz zu diesem Thema ist gering, die Diskussion aller Optionen würde ein eigenes Heft füllen. So erläutern Wendl-Söldner et al. am Beispiel der Hüftfraktur das Gerinnungsmanagement in der perioperativen Phase. Mit bis zu $60 \%$ ist das Delir wohl eine der häufigsten Komplikationen bei älteren Patienten nach chirurgischen Eingriffen [3]. Da die therapeutischen Möglichkeiten in der Behandlung des Delirs weiterhin unbefriedigend sind und die Umsetzung von präventiven Maßnahmen im heutigen Klinikalltag schwierig ist, ist die Versuchung groß, zur pharmakologischen Prävention zu greifen. Der vorliegende Review gibt einen Überblick über mögliche Regime, zeigt aber auch deutlich die Grenzen des heutigen Wissens auf. Luger at al. diskutieren in ihrem Beitrag die Wahl der Anästhesie. Nach einem operativen Eingriff bedürfen viele geriatrische Patienten über einen gewissen Zeitraum einer intensivmedizinischen Betreuung. Oxygenierung, Ernährung, Analgesie und intensives Monitoring tragen zur erfolgreichen Behandlung bei operativ versorgten geriatrischen Patienten bei und die Besonderheiten dieser Patientengruppe müssen beachtet werden. Trotz sehr hoher Prävalenzzahlen der Malnutrition von bis $60 \%$ wird der Stellenwert der pe- rioperativen Ernährungstherapie häufig unterschätzt [4]. Schulz et al. beleuchten das Problem der Malnutrition und geben einen Überblick über die therapeutischen Möglichkeiten.

Wir hoffen, mit unserem Themenschwerpunkt dem Trend der Zeit nach einer verstärkten Einbindung der Geriatrie in den Akutbereich gerecht geworden zu sein und Ihnen in einigen Fragestellungen hilfreich sein zu können. In vielen Fragen ist die Evidenz noch ungenügend. So sollen die Beiträge die Diskussion anregen und auch Ansporn sein, Studien zu diesen Fragestellungen zu initiieren.

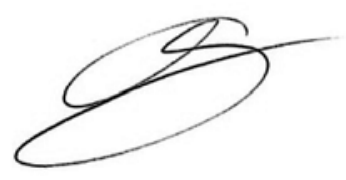

\section{Gosch}

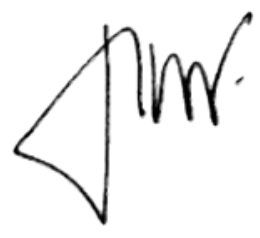

H.J. Heppner

\section{Korrespondenzadressen}

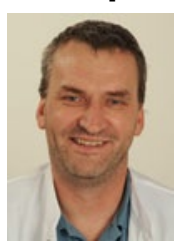

Dr. M. Gosch

Abteilung für Geriatrie und Innere Medizin, Hospital Hochzirl Hochzirl 1, 6170 Zirl

Österreich

markus.gosch@tilak.at

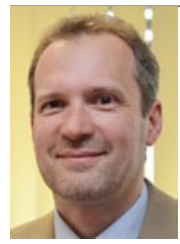

H.J. Heppner Geriatrische Klinik HELIOS Klinikum Schwelm hjheppner@online.de

\section{Einhaltung ethischer Richtlinien}

Interessenkonflikt. M. Gosch und H. J. Heppner geben an, dass kein Interessenkonflikt besteht.

Dieser Beitrag beinhaltet keine Studien an Menschen oderTieren.

\section{Literatur}

1. Berger E. (2008) The graying of America: the impact of aging baby boomers on emergency departments. Ann Emerg Med 51:288-90

2. Das Informationssystem der Gesundheitsberichterstattung des Bundes. http://www.gbe-bund.de. Zugegriffen: 08. Januar 2014

3. Krankenhausgesellschaft zur OECD-Studie: Falsche Schlussfolgerungen. www.aerzteblatt.de/ nachrichten/53987/Krankenhausgesellschaft-zurOECD-Studie-Falsche-Schlussfolgerungen. Zugegriffen: 09. April 2013.

4. Inouye SK (2006) Delirium in older adults. N Engl J Med 354:1157-1165

5. Volkert D, Sieber CC (2011) Mangelernährung in der Geriatrie. Aktuel Ernaehrungsmed 36:175-190 